\title{
On the design of Robust tube-based MPC for tracking *
}

\author{
D. Limon ${ }^{*}$ I. Alvarado* T. Alamo* E. F. Camacho* \\ * Dpto. de Ingeniería de Sistemas y Automática, Universidad de \\ Sevilla, Avda de los Descubrimeintos s/ $n$ 41092, Sevilla (limon, \\ alvarado, alamo, camacho@cartuja.us.es).
}

\begin{abstract}
This paper deals with the design procedure of the recently presented robust MPC for tracking of constrained linear systems with additive disturbances. This controller is based on nominal predictions and it is capable to steer the nominal predicted trajectory to any target admissible steady state, that is retaining feasibility under any set point change. By means of the notion of tube of trajectories, robust stability and convergence is achieved.

The controller formulation has some parameters which provides extra degrees of freedom to the design procedure of the predictive controller. These allow to deal with control objectives such as disturbance rejection, output offset prioritization or enlargement of the domain of attraction. In this paper, output prioritization method, LMI based design procedures and algorithms for the calculation of invariant sets are presented. The proposed enhanced design of the MPC is demonstrated by an illustrative example.
\end{abstract}

\section{INTRODUCTION}

Model predictive control (MPC) is a control technique capable to deal with hard constraints of the system and the optimization of a performance index. This is achieved by posing the control problem as a mathematical programming problem and applying the optimal solution in a receding horizon manner. This controller typically requires a terminal state penalization and constraint, in order to ensure closed loop stability. The stabilizing ingredients and the finite prediction horizon make that the predictive controller may loose feasibility under set point changes.

Recently, a novel Robust MPC for tracking has been presented (Alvarado et al. [2007]). The main characteristics of this controller are: (i) adds an artificial steady state as a decision variable (artificial steady state) (ii) uses an invariant set for tracking as a terminal constraint (iii) considers a cost function penalizing the error w.r.t the artificial steady state and an additional term penalizing the deviation between the artificial steady state and the target steady state (iv) it is based on the tube-based MPC controller (Mayne et al. [2005]). This controller, under mild assumptions, can steer the uncertain system in an admissible evolution to a neighborhood of any admissible steady state.

The robust MPC for tracking has some parameters to be tuned, which provides extra degrees of freedom to be exploited according to the control objectives. This allows us to deal with disturbance rejection, enlargement of the domain of attraction and output tracking prioritization when the set point is not admissible.

In this paper, the effect of each parameter of the MPC is analysed and procedures for their selection are presented.

\footnotetext{
* The authors acknowledge MCYT-Spain for funding this work (contracts DPI2007-66718-C04-01 and DPI2005-04568)
}

First, it is shown how to design the proposed MPC to ensure the evolution to an optimized steady state when the target is not admissible. Then the robust local controller is designed to minimize the effect of the disturbances on the state evolution and hence to enlarge de domain of attraction of the MPC. This can be posed as a set of linear matrix inequalities which solution provides the control gain. Finally, existing results on the computation of an approximation to the minimal robust positively invariant set are specialized for disturbances that can be represented as a zonotope (an affine mapping of a unitary box).

The paper is structured as follows: In $\S 2$ the problem to solve is described and in $\S 3$ the robust MPC for tracking is presented, in $\S 4$ the design procedure is introduced, in $\S 5$ the tool that minimize the invariant set. The paper finishes with an illustrative example in section 5 and some conclusions.

Notation: A definite positive matrix $T$ is denoted as $T>0$ and $T>P$ denotes that $T-P>0$. For a given symmetric matrix $P>0,\|x\|_{P}$ denotes the weighted Euclidean norm of $x$, i.e. $\|x\|_{P}=\sqrt{x^{\top} P x}$. $(a, b) \triangleq\left[a^{\top}, b^{\top}\right]^{\top}$. Consider $a \in \mathbb{R}^{n_{a}}, b \in \mathbb{R}^{n_{b}}$, and set $\Gamma \subset \mathbb{R}^{n_{a}+n_{b}}$, then projection operation is defined as $\operatorname{Proj}_{a}(\Gamma)=\left\{a \in \mathbb{R}^{n_{a}}: \exists b \in \mathbb{R}^{n_{b}},(a, b) \in \Gamma\right\}$. Given two sets $\mathcal{U}$ and $\mathcal{V}$, such that $\mathcal{U} \subset \mathbb{R}^{n}$ and $\mathcal{V} \subset \mathbb{R}^{n}$, the Minkowski sum is defined by $\mathcal{U} \oplus \mathcal{V} \triangleq\{u+v \mid u \in \mathcal{U}, v \in \mathcal{V}\}$, the Pontryagin set difference is: $\mathcal{U} \ominus \mathcal{V} \triangleq\{u \mid u \oplus \mathcal{V} \subseteq \mathcal{U}\}$. For a given matrix $M \in \mathbb{R}^{n \times m}$ and a set $\mathcal{V} \subset \mathbb{R}^{m}$, the set $M \mathcal{V} \subset \mathbb{R}^{n}$ denotes the set $\{y=M v, v \in \mathcal{V}\}$. For a given $\lambda, \lambda \mathcal{X}=\{\lambda x: x \in \mathcal{X}\}$. Let $\mathbf{t}$ be a generic vector defined as $\mathbf{t} \triangleq\{t(0), t(1), \ldots\}$. A set is a $\mathrm{C}$ set if is compact, convex not empty. A matrix $\mathbf{0}_{n, m} \in \mathbb{R}^{n \times m}$ denotes a matrix of zeros. The set of vertexes of a given $\mathrm{C}$ set $\Gamma$ is denoted 
as $\operatorname{vert}(\Gamma)$. The set $\mathcal{B}^{N} \subset \mathbb{R}^{N}$ denotes the unitary ball $\mathcal{B}^{N}=\left\{b \in \mathbb{R}^{N}:\|b\|_{\infty} \leq 1\right\}$.

\section{PROBLEM DESCRIPTION}

Consider the following uncertain discrete-time LTI system:

$$
x^{+}=A x+B u+w, \quad y=C x+D u
$$

subject to following constraints:

$$
x \in \mathcal{X} \subset \mathbb{R}^{n} \quad u \in \mathcal{U} \subset \mathbb{R}^{m}
$$

and disturbances set:

$$
w \in \mathcal{W} \subset \mathbb{R}^{n}
$$

where: $x$ is the current state, $u$ is the current control action, $x^{+}$is the successor state, $w$ is an unknown state disturbance, $y \in \mathbb{R}^{p}$ is the current measured output, and $(A, B, C, D) \in \mathbb{R}^{n \times n} \times \mathbb{R}^{n \times m} \times \mathbb{R}^{p \times n} \times \mathbb{R}^{p \times m} . \mathcal{W}$, is a compact, convex not empty set and $\mathcal{X}, \mathcal{U}$ are polyhedral and polytopic sets respectively.

Let $\phi(i ; x, \mathbf{u}, \mathbf{w})$ denote the solution of (1) at time $i$ if the initial state is $x$ and the control and disturbance sequences are, respectively, $\mathbf{u}$ and $\mathbf{w}$.

The overall objective is to stabilize the constrained system and steer the state to a neighborhood of the setpoint fulfilling the constraints for any possible disturbance.

The following standing assumption is made: Assumption 1. The couple $(A, B)$ is controllable.

\section{ROBUST MPC FOR TRACKING}

For the sake of a clear and self-contained exposition of the contributions of the paper, the robust MPC for tracking is succinctly presented in this section. A more detailed explanation of the controller can be found in (Alvarado et al. [2007], Alvarado. [2007])

\subsection{Tube of trajectories}

The proposed controller is based on the response of the nominal system, obtained from (1) by neglecting the disturbances $w$. The nominal system is described by:

$$
\bar{x}^{+}=A \bar{x}+B \bar{u}, \quad \bar{y}=C \bar{x}+D \bar{u}
$$

Choosing an initial state $\bar{x}$ and a control sequence $\overline{\mathbf{u}}$ yields a state sequence $\overline{\mathbf{x}}$ obtained by solving $(4)\left(\bar{x}_{i}=\bar{\phi}(i ; \bar{x}, \overline{\mathbf{u}})\right)$. To counteract the disturbances it is desirable to force the trajectory to lie close to the nominal trajectory; this can be done by choosing the control u to satisfy:

$$
u=\bar{u}+K e \quad e \triangleq(x-\bar{x})
$$

where $e$ denotes the error between the state and the state of the nominal system. The error $e$ dynamics is given by

$$
e^{+}=A_{K} e+w ; \quad A_{K}=(A+B K)
$$

If matrix $A_{K}$ is Hurwitz then there exists a robust positively invariant set $\mathcal{Z}$ (Kolmanovsky and Gilbert [1998], Rakovic et al. [2005]) for the system (6) that satisfies

$$
A_{K} \mathcal{Z} \oplus \mathcal{W} \subseteq \mathcal{Z}
$$

The invariance property allows to state the notion of tube of trajectories given in the following proposition:
Proposition 1. (Mayne et al. [2005]). If the initial real and nominal system states, satisfy $e(0)=x(0)-\bar{x}(0) \in \mathcal{Z}$, then $x(i) \in \bar{x}(i) \oplus \mathcal{Z} \quad \forall i \in \mathbb{N}$, for all disturbance sequences $\mathbf{w}$ such that $w(i) \in \mathcal{W} \quad \forall i=1,2, \ldots$

From this proposition it is inferred that if the nominal control actions are calculated to ensure that the nominal predicted states and inputs satisfy the following tighter constraints

$$
\overline{\mathcal{X}}=\mathcal{X} \ominus \mathcal{Z} \quad \overline{\mathcal{U}}=\mathcal{U} \ominus K \mathcal{Z}
$$

then the system controlled by (5) maintains all the possible trajectories admissible, that is satisfying the constraints on states and inputs for all possible uncertainty (Mayne et al. [2005]).

\subsection{Set point characterization and invariant set for tracking}

In this section, a suitable parametrization of the steady states and inputs for the nominal system is presented. Consider a given output target $y_{t}$, then any steady state of the nominal system $z_{s}=\left(x_{s}, u_{s}\right)$ associated to this setpoint must satisfy the following equation

$$
\left[\begin{array}{ccc}
A-I_{n} & B & 0_{n, 1} \\
C & D & -I_{p}
\end{array}\right]\left[\begin{array}{l}
x_{s} \\
u_{s} \\
y_{t}
\end{array}\right]=\left[\begin{array}{l}
0_{n, 1} \\
0_{p, 1}
\end{array}\right]
$$

Because of the pair $(A, B)$ is stabilizable, the solution to this problem can be parameterized as

$$
z_{s}=M_{\theta} \theta \quad y_{t}=N_{\theta} \theta
$$

where $\theta \in \mathbb{R}^{n_{\theta}}$ is a parameter vector which characterizes any solution, and $M_{\theta}$ and $N_{\theta}$ are suitable matrices (Limon et al. [2008]).

The existence of constraints (7) limits the set of reachable steady states and inputs. The set of admissible steady states is denoted as $\mathcal{X}_{s}$ and it is a polyhedron given by $\mathcal{X}_{s}=\left\{x_{s} \in \overline{\mathcal{X}}: \exists u_{s} \in \mathcal{U} \mid\left(A-I_{n}\right) x_{s}+B u_{s}=0_{n, 1}\right\}$.

Now, the notion of invariant set for tracking is presented Definition 1. Let $x^{e}$ be the extended state $(x, \theta) \in$ $\mathbb{R}^{n+n_{\theta}}$, let $K_{\Omega}$ be a control gain such that $A+B K_{\Omega}$ is Hurwitz and let $K_{\theta}$ be given by $K_{\theta}=\left[\begin{array}{ll}-K_{\Omega} & I_{m}\end{array}\right] M_{\theta}$. Then, a set $\Omega_{t}^{e} \subset \mathbb{R}^{n+n_{\theta}}$ is an invariant set for tracking admissible for $(7)$, if for all $(x, \theta) \in \Omega_{t}^{e}$, then $x \in \overline{\mathcal{X}}$, $K_{\Omega} x+K_{\theta} \theta \in \overline{\mathcal{U}}$ and $\left(\left(A+B K_{\Omega}\right) x+B K_{\theta} \theta, \theta\right) \in \Omega_{t}^{e}$.

The role of the presented parametrization is to simplify the proposed predictive controller as well as the calculation of invariant sets for tracking.

\subsection{Predictive controller}

The main objective of this controller is to robustly steer the output system to a (neighborhood of) a target $y_{t}$. It is assumed that this target can be characterized by a parameter vector $\theta$ by means of (9) (i.e. it is a possible steady output for the nominal system).

This controller, as the tube-based controller (Mayne et al. [2005]), considers as decision variables the initial nominal state $\bar{x}$ and the sequence of future nominal control actions $\overline{\mathbf{u}}$. In order to deal with the set point change problem an 
artificial steady state and input $\left(\bar{x}_{s}, \bar{u}_{s}\right)=M_{\theta} \bar{\theta}$ is considered as decision variable. These variables are calculated to minimize the following nominal prediction based cost function:

$$
\begin{aligned}
V_{N}(x, \theta ; \overline{\mathbf{u}}, \bar{x}, \bar{\theta}) & =\sum_{i=0}^{N-1}\left(\left\|\bar{x}(i)-\bar{x}_{s}\right\|_{Q}^{2}+\left\|\bar{u}(i)-\bar{u}_{s}\right\|_{R}^{2}\right) \\
& +\left\|\bar{x}(N)-\bar{x}_{s}\right\|_{P}^{2}+\|\bar{\theta}-\theta\|_{T}^{2}
\end{aligned}
$$

where $\bar{x}(i)=\bar{\phi}(i ; \bar{x}, \overline{\mathbf{u}})$ and $\left(\bar{x}_{s}, \bar{u}_{s}\right)=M_{\theta} \bar{\theta}$. The current state $x$ and the target operation point given by $\theta$ are parameters of the cost function.

This cost penalizes the deviation between the predicted trajectory and the artificial steady state along the horizon $N$. The deviation between the artificial steady conditions (given by $\bar{\theta}$ ) and the target one (given by $\theta$ ) is penalized by means of the so-called offset cost $\|\bar{\theta}-\theta\|_{T}^{2}$.

The cost function must be minimized considering the constraints derived from stability and admissibility conditions, leading to the following quadratic problem $\mathcal{P}_{N}(x, \theta)$ :

$$
\begin{aligned}
\min _{\bar{x}, \overline{\mathbf{u}}, \theta} & V_{N}(x, \theta ; \overline{\mathbf{u}}, \bar{x}, \bar{\theta}) \\
\text { s.t. } & \bar{x} \in x \oplus(-\mathcal{Z}) \\
& \bar{x}(i) \in \overline{\mathcal{X}}, \quad i=0, \cdots, N \\
& \bar{u}(i) \in \overline{\mathcal{U}} \quad i=0, \cdots, N-1 \\
& (\bar{x}(N), \bar{\theta}) \in \Omega_{t}^{e}
\end{aligned}
$$

where: $\overline{\mathcal{X}}=\mathcal{X} \ominus \mathcal{Z}$ and $\overline{\mathcal{U}}=\mathcal{U} \ominus K \mathcal{Z}$.

$\mathcal{P}_{N}(x, \theta)$ is solved online to yield an optimal initial state $\bar{x}^{*}(x, \theta)$, an optimal nominal control sequence $\overline{\mathbf{u}}^{*}(x, \theta)=$ $\left\{\bar{u}^{*}(0, x, \theta), \bar{u}^{*}(1, x, \theta), \ldots, \bar{u}^{*}(N-1, x, \theta)\right\}$ and the optimal artificial steady conditions parameter $\theta^{*}(x, \theta)$. From this, the control applied to the plant is given by:

$$
k_{N}(x, \theta)=\bar{u}^{*}(0, x, \theta)+K\left(x-\bar{x}^{*}(x, \theta)\right)
$$

Notice that the set of constraints that defines the feasibility region of $\mathcal{P}_{N}(x, \theta)$ does not depend on $\theta$ (that is, the target operating point), but only on the current state $x$ and the decision variables. Therefore, this feasibility region only depends on the current state $x$. Thus, defining $\overline{\mathcal{X}}_{N} \subset \mathbb{R}^{n}$ as the set of the admissible nominal initial states $\bar{x}$ such that (13), (14) and (15) hold, the set of feasible states is given by $\mathcal{X}_{N}=\overline{\mathcal{X}}_{N} \oplus \mathcal{Z}$.

\subsection{Stabilizing conditions of the controller}

In order to ensure robust stability of the closed loop system, the following sufficient conditions on the defining ingredients are assumed:

Assumption 2. The matrices $Q, R, T, P, K, K_{\Omega}$, and sets $\Omega_{t}^{e}$ and $Z$ fulfil:

- $Q>0$ and $R>0$

- There exists a constant $\sigma>0$ such that $\sigma T \geq M_{x}^{T} M_{x}$, where $M_{x}=\left[I_{n}, \mathbf{0}_{n}\right] M_{\theta}$.

- The gain matrix $K$ is such that $A+B K$ is Hurwitz.

- The pair of matrices $K_{\Omega}$ and $P$ are such that $A+B K_{\Omega}$ is Hurwitz and $P>0$ where

$$
P-\left(A+B K_{\Omega}\right)^{\top} P\left(A+B K_{\Omega}\right)=Q+K_{\Omega}^{\top} R K_{\Omega}
$$

- Set $\mathcal{Z} \subset X$ is an admissible robust positively invariant set such that $(A+B K) \mathcal{Z} \oplus W \subseteq Z$ and $K \mathcal{Z} \subset U$.

- $\Omega_{t}^{e}$ is an invariant set for tracking (as large as possible) for the nominal system (4) subject to the constraints $\overline{\mathcal{X}}$ and $\overline{\mathcal{U}}$ and using as control gain matrix $K_{\Omega}$.

Now it is possible to establish the following result:

Theorem 1. (Alvarado et al. [2007]). Consider system (1) subject to the constraints (2) and such that verifies Assumptions 1 and 2 . Let $k_{N}(x, \theta)$ be the control law resulting from the solution of the optimal problem $\mathcal{P}_{N}(x, \theta)$. Let $Y_{t}$ be the set of reachable target outputs given by

$$
Y_{t} \triangleq\left\{y=N_{\theta} \theta: M_{\theta} \theta \in \operatorname{Proj}_{x}\left(\Omega_{t}^{e}\right) \times \overline{\mathcal{U}}\right\}
$$

Then $\forall x(0) \in \mathcal{X}_{N}$ and a target $y_{t} \in Y_{t}$ the closed loop system fulfils the constraints (2) along its evolution and converges asymptotically to $x_{s} \oplus \mathcal{Z}$.

The proof of this theorem can be found in (Alvarado. [2007])

\subsection{Cancellation of the output offset}

The proposed controller is able steer the system state to a neighborhood of the target steady state, that is $x_{s} \oplus \mathcal{Z}$. If the disturbances are decaying, the system converges asymptotically to the target, but if the disturbance tends to a steady value, the the closed loop system presents offset on the outputs, i.e. the error $y(k)-y_{t}$ tends to a constant value.

This can be cancellated by the following methods. One possible technique is augmenting the model of the system with integrating disturbances to remove offset (Pannocchia [2004]); a linear control law ensures robust offset-free control at expense of enlarging three times the order of the plant which makes the controller design and calculation more difficult.

A second method proposed by the authors in (Alvarado et al. [2007]), achieves the offset free without augmenting the plant, but adding an outer loop consisting of a disturbance estimator and a set point correction given by

$$
\hat{y}_{t}(k)=y_{t}-(C+D K)\left(I_{n}+(A+B K)\right)^{-1} \hat{w}(k)
$$

where $\hat{w}(k)$ is the estimated disturbance. The estimator dynamics should be adjusted according the dynamics of the disturbance signal and the closed loop system.

\section{SYNTHESIS OF THE PROPOSED CONTROLLER}

The presented predictive controller is designed by picking the defining parameters (horizon, matrices and sets) fulfilling Assumption 2. Since this does not fix the parameters, these can be chosen according to control objectives, such as closed loop performance, disturbance rejection, domain of attraction, etc.

Matrices $Q$ and $R$ define the stage cost which is closed loop performance index. Control horizon $N$ is typically chosen as large as possible, since a large $N$ provides a larger domain of attraction and an enhancement of the closed loop performance. However, the dimension of the problem grows with $N$, and hence requires a larger computational time. Therefore, a tradeoff must be achieved. 
In this section, the chosen of the extra parameters specific of the proposed predictive controller: the matrices $T, K$, $K_{\Omega}, P$ and the sets $\mathcal{Z}$ and $\Omega_{t}^{e}$.

\subsection{The parameter $T$}

This matrix defines the weight of the tracking error cost $\|\bar{\theta}-\theta\|_{T}^{2}$ in the cost function. The effects of this parameter on the closed loop system are the following :

- Transient dynamics and set point filtering: if matrix $T$ is chosen to penalize more heavily the tracking error cost, then the convergence of $\bar{\theta}$ to $\theta$ is made faster, and hence the transient of the closed loop system to the target.

- Local optimality: it is well known that MPC can be designed to be locally infinite horizon optimal (for the nominal system), however the addition of the artificial steady state and input makes that this property does not hold in the presented controller. The optimality loss can be arbitrarily reduce by picking a large enough matrix $T$.

- Offset minimization: this is a remarkable property of the controller (Alvarado. [2007]). Consider that the target $y_{t}$ is not reachable that is, the desired output $y_{t}$ does not fulfil any of the constraints, i.e. $M_{\theta} \theta \notin \Omega_{t} \times \overline{\mathcal{U}}, \forall \theta: y_{t}=N \theta$. Under these conditions, the target can not be reached and then, the output will present offset.

In this case the proposed controller drives the system to the neighborhood $\tilde{x}_{s} \oplus \mathcal{Z}$, where the steady state $\tilde{x}_{s}$ (given by the parameter $\tilde{\theta}$ ) is the one which minimizes the offset cost. That is

$$
\begin{array}{r}
\tilde{\theta}=\arg \min _{\bar{\theta}}\|\bar{\theta}-\theta\|_{T}^{2} \\
M_{\theta} \bar{\theta} \in \Omega_{t} \times \overline{\mathcal{U}}
\end{array}
$$

Therefore, matrix $T$ allows us to prioritize some outputs (by weighting more heavily its corresponding term in matrix $T$ ) to achieve a minimum offset on this outputs. See that this prioritization does not vary with the scaling of matrix $T$, and hence the effects previously presented can be simultaneously considered in the design of $T$.

Then the sensible way to design $T$ is firstly pick the structure according to the offset minimization, and then, scale the matrix to achieve a quick transient with a small enough optimality loss. Finally notice that since the value of $T$ is independent of the rest of parameters, this can be tuned online maintaining the stabilizing properties.

\subsection{The parameters $K_{\Omega}, P$ and $\Omega_{t}^{e}$}

The terminal cost and terminal set strongly depend on the control gain $K_{\Omega}$. This can be designed according the following aspects: from a performance point of view, it is desirable that the terminal cost is taken as the cost-to-go, which can be ensured if $K_{\Omega}$ is chosen as the LQ regulator gain. From a domain of attraction viewpoint, it is desirable to take $K_{\Omega}$ to achieve a large terminal set $\Omega_{t}^{e}$.

In this case, the large size of the invariant set for tracking provides a large domain of attraction $\mathcal{X}_{N}$, even for small values of the control horizon. Then, the most sensible way to pick $K_{\Omega}$ is the Linear Quadratic Regulator and the matrix $P$ as the solution of the Riccati equation.

The invariant set for tracking $\Omega_{t}^{e}$ can be taken as a polyhedral set which approximates arbitrarily to the maximal invariant set for tracking thanks to a simple procedure (Limon et al. [2008]. This provides a set of reachable targets $Y_{t}$ practically equal to the maximal one (i.e. the difference between both sets can be made arbitrarily small).

\subsection{The parameter $K$}

This parameter has an important role in the proposed controller since this control gain is used to compensate the deviation from the nominal predictions in the controller (16), and therefore, it characterizes the dynamics of the closed loop system in the presence of disturbances. This makes that $K$ is chosen according to a robustness or disturbance rejection criterium.

In this paper, we consider as robustness criterium the minimization of the minimum admissible robust positively invariant set. Thus, $K$ is chosen to: (i) ensure the existence of an admissible robust positively invariant set $\mathcal{Z}$ such that the sets $X \ominus \mathcal{Z}$ and $U \ominus K \mathcal{Z}$ are not empty sets; (ii) minimize the size of $\mathcal{Z}$. This criterium provides a larger domain of attraction and a larger set of reachable targets as well as minimizes the effect of the disturbance on the trajectory of the system.

Consider w.l.o.g. that set $\mathcal{X}=\left\{x:\left|h_{i}^{\top} x\right| \leq 1, i=\right.$ $\left.1, \cdots, n_{r x}\right\}$ and set $\mathcal{U}=\left\{u:\left|\ell_{j}^{\top} u\right| \leq 1, j=1, \cdots, n_{r u}\right\}$. Then, the synthesis problem to solve is to calculate the control law $u=K x$ such that the size of the ellipsoid $\mathcal{E}(P, 1)=\left\{x \in \mathbb{R}^{n}: x^{\top} P x \leq 1\right\}$ is minimized fulfilling that:

(i) $\mathcal{E}(P, 1)$ is a robust invariant set for system (6). This condition can be reformulated as follows:

$$
\left(x^{+}\right)^{\top} P x^{+} \leq 1, \quad \forall x \in \mathcal{E}(P, 1), \forall w \in \mathcal{W}
$$

Applying the S-procedure, and considering the convexity with relation to $w$, equation (17) is satisfied if exists $\lambda \geq 0$ such that:

$$
\begin{aligned}
& \left(\left(A_{K}\right) x+w\right)^{\top} P\left(\left(A_{K}\right) x+w\right)+\lambda\left(1-x^{\top} P x\right)<1 \\
& A_{K}=A+B K \quad \forall x \in \mathbb{R}^{n}, \forall w \in \operatorname{vert}(\mathcal{W})
\end{aligned}
$$

where $\operatorname{vert}(\mathcal{W})$ denotes the set of vertexes of $\mathcal{W}$. This can be rewritten as:

$$
\left[\begin{array}{cc}
\lambda P-(A+B K)^{\top} P(A+B K) & -(A+B K)^{\top} P w \\
-w^{\top} P(A+B K) & 1-\lambda-w^{\top} P w
\end{array}\right]>0
$$

$\forall w \in \operatorname{vert}(\mathcal{W})$

(ii) For all $x \in \mathcal{E}(P, 1)$, the control law $\left|\ell_{j}^{\top} K x\right| \leq \rho_{j}$ for all $j=1, \cdots, n_{r u}$ and $\rho_{j} \in(0,1]$. The role of the parameter $\rho_{j}$ is to restrict the set of admissible control inputs to guarantee a given control range of the the MPC controller i.e. the set $\overline{\mathcal{U}}=\mathcal{U} \ominus K \mathcal{Z}$ has not empty interior.

This condition can be posed as:

$$
\ell_{j}^{\top} K P^{-1} K^{\top} \ell_{j} \leq \rho_{j}^{2}, j=1, \ldots, n_{r u}
$$

Applying the Schur complement, this yields to

$$
\left[\begin{array}{cc}
\rho_{j}^{2} & \ell_{j}^{\top} K \\
K^{\top} \ell_{j} & P
\end{array}\right]>0, j=1, \ldots, n_{r u}
$$


(iii) For all $x \in \mathcal{E}(P, 1),\left|h_{i}^{\top} x\right| \leq 1$. Considering similar arguments to the previous fact, this condition is equivalent to

$$
\left[\begin{array}{cc}
1 & h_{i}^{\top} \\
h_{i} & P
\end{array}\right]>0, i=1, \ldots, n_{r x}
$$

In order to minimize the size of the ellipsoid $\mathcal{E}(P, 1)$, a suitable measure of this set must be chosen. In this paper, we propose as measure a parameter $\gamma>0$ such that $\mathcal{E}(P, 1) \subseteq \sqrt{\gamma} \mathcal{X}$. Therefore, minimize the size of $\mathcal{E}(P, 1)$ is posed as minimizing the parameter $\gamma$. Obviously, admissibility of the solution requires that $\gamma \leq 1$.

Applying standard operations of LMIs (Boyd et al. [1994]), the proposed synthesis procedure can be formulated as the solution of the following convex optimization problem:

$$
\begin{aligned}
& \min _{Y, W, \gamma} \quad \gamma \\
& \text { s.a. } \\
& {\left[\begin{array}{ccc}
\lambda W & * & * \\
0 & 1-\lambda & * \\
A W+B Y & w & W
\end{array}\right]>0, \quad \forall w \in \operatorname{vert}(\mathcal{W})} \\
& {\left[\begin{array}{cc}
\rho_{i}^{2} & * \\
Y^{\top} \ell_{i} & W
\end{array}\right]>0, i=1, \ldots, n_{r u}} \\
& {\left[\begin{array}{cc}
\gamma & * \\
W h_{i} & W
\end{array}\right]>0, i=1, \ldots, n_{r x}}
\end{aligned}
$$

for a given $\lambda \geq 0$. If feasible, the ellipsoid is given by $P=W^{-1}$ and the control gain is $K=Y W^{-1}$. It is worth remarking that any robust criterium that can be posed as LMIs can be added to this synthesis problem.

\subsection{Calculation of the robust invariant set $\mathcal{Z}$}

Once the control gain $K$ is designed, an admissible robust positively invariant (RPI) set (as small as possible) must be calculated. See that the proposed synthesis of $K$ ensures the existence of this set. It would be desirable to compute the minimum robust positively invariant set (mPRI) $F_{\infty}$ (Kolmanovsky and Gilbert [1998], given by

$$
F_{s}=\bigoplus_{k=0}^{s}(A+B K)^{k} \mathcal{W}
$$

when $s$ tends to infinity. Unfortunately, $F_{\infty}$ can only be calculated for some special cases, such as, when a deadbeat control law is used.

In the recent paper (Rakovic et al. [2005], a procedure for the determination of an invariant approach of $F_{\infty}$ is presented. This allows one to compute a RPI $\mathcal{Z}$ such that $F_{\infty} \subseteq \mathcal{Z} \subseteq F_{\infty} \oplus \epsilon \mathcal{B}^{n}$ for a given bound of the absolute error $\epsilon$. To this aim, the following functions are calculated

$$
\begin{aligned}
& \alpha(s)=\min \alpha:(A+B K)^{s} \mathcal{W} \subseteq \alpha \mathcal{W} \\
& \beta(s)=\min \beta: F_{s} \subseteq \beta \mathcal{B}^{n}
\end{aligned}
$$

These values are calculated by solving a number of Linear Programming problems. If a large enough value of $s$ is taken such that $(1-\alpha(s))^{-1} \alpha(s) \beta(s) \leq \epsilon$ then the set $\mathcal{Z}=(1-\alpha(s))^{-1} F_{s}$ is an approximation of $F_{\infty}$ with an error bound less than $\epsilon$.
From a practical point of view, it might be desirable to calculate the approximation for a relative error bound, which does not require an a priori estimation of the size of the invariant set to choose the error bound. This is stated in the following lemma.

Lemma 1. Let $s$ be a positive real number such that

$$
\alpha(s) \leq \frac{\lambda}{1+\lambda}
$$

for a given relative error bound $\lambda \in(0,1)$. Then the set $\mathcal{Z}=(1-\alpha(s))^{-1} F_{s}$ is a RPI such that

$$
F_{\infty} \subseteq \mathcal{Z} \subseteq(1+\lambda) F_{\infty}
$$

On the other hand, we specialize this result for the case that the uncertainty set is a zonotope of the form

$$
\mathcal{W}=H \mathcal{B}^{n} \oplus w_{0}
$$

where $H \in \mathbb{R}^{n \times n}$ is non singular. This is motivated because this class of set is frequently used to bound additive uncertainties in practice. For this class of uncertainties we can state the following lemma.

Lemma 2. Consider a set $\mathcal{W}=H \mathcal{B}^{n} \oplus w_{0}$ where $H$ is a non singular matrix. Denote $(A+B K)$ as $A_{K}$ and define the matrix $H_{z}(s)=\left[A_{K}^{s-1} H, A_{K}^{s-2} H, \cdots, H\right]$. Then

$$
\begin{aligned}
\left\|H^{-1} A_{K}^{s} H\right\|_{\infty} & =\min \alpha: A_{K}^{s} H \mathcal{B}^{n} \subseteq \alpha H \mathcal{B}^{n} \\
\left\|H_{z}(s)\right\|_{\infty} & =\min \beta: \bigoplus_{k=0}^{s-1} A_{K}^{k} H \mathcal{B}^{n} \subseteq \beta \mathcal{B}^{n}
\end{aligned}
$$

Based on this lemma, an approximation to the mRPI with a relative error boud $\lambda$ for the case of zonotopic uncertainties is presented in the following lemma.

Lemma 3. Let $s$ be such that

$$
\left\|H^{-1}(A+B K)^{s} H\right\|_{\infty} \leq \frac{\lambda}{1+\lambda}
$$

for a given relative error bound $\lambda \in(0,1)$ and denote $\hat{\alpha}(s)=\left\|H^{-1}(A+B K)^{s} H\right\|_{\infty}$. Then the zonotope $\mathcal{Z}$ given by

$$
\mathcal{Z}=(1-\hat{\alpha}(s))^{-1} H_{z}(s) \mathcal{B}^{s n} \oplus\left(I_{n}-(A+B K)\right)^{-1} w_{0}
$$

is a RPI such that $F_{\infty} \subseteq \phi_{K} \subseteq(1+\lambda) F_{\infty}$.

The proofs of these lemmas can be found in ([Alvarado., 2007, Chapter 6].

The zonotope expression of the invariant set makes easier the calculation of the linear mapping and Pontryagin difference, and consequently the calculation of the polytopes $\mathcal{X} \ominus \mathcal{Z}$ and $\mathcal{U} \ominus K \mathcal{Z}$. However, constraint (12) requires the calculation of the set of inequalities (hyperplanes) that define $\mathcal{Z}$. A zonotope is a compact expression of a polytope with a number of facets that grows exponentially with the dimension of the unitary box $(s n)$. Although there exists specialized algorithms to obtain the facets, these are only tractable for a reduced dimension of the zonotope. In ([Alvarado., 2007, Chapter 6]) can be found several practical procedures for this calculation.

\section{ILLUSTRATIVE EXAMPLE}

Consider a constrained sampled double integrator 


$$
\begin{aligned}
x^{+} & =\left[\begin{array}{ll}
1 & 1 \\
0 & 1
\end{array}\right] x+\left[\begin{array}{ll}
0 & 0.5 \\
1 & 0.5
\end{array}\right] u+w \\
y & =\left[\begin{array}{ll}
0 & 1
\end{array}\right] x .
\end{aligned}
$$

where the disturbances are bounded in $w \in \mathcal{W}=0.1 \mathcal{B}^{2}$. The system must fulfil the following constraints: $\left|x_{1}\right| \leq 5$, $\left|x_{2}\right| \leq 5,\left|u_{1}\right| \leq 0.3,\left|u_{2}\right| \leq 0.3$.

The objective is to show the proposed procedures to find a suitable robust controller gain $K$ to ensure the admissibility of the tube-based predictive controller, i.e. ensuring that $\mathcal{X} \ominus \mathcal{Z}$ and $\mathcal{U} \ominus K \mathcal{Z}$ are not empty sets.

To test the proposed synthesis method, we design two matrices, $K_{L Q R}$ and $K_{o p}$. The first matrix is obtained as the LQR gain for $Q=I_{2}$ and $R=10 I_{2}$. The second matrix is designed using the proposed method with a value of $\rho=0.48$ in order to get the same set $K \mathcal{Z}$ as in the previous case. Figure 1 shows the approximated RPI for both gains. It can be seen that the proposed method provides a smaller set $\mathcal{Z}$
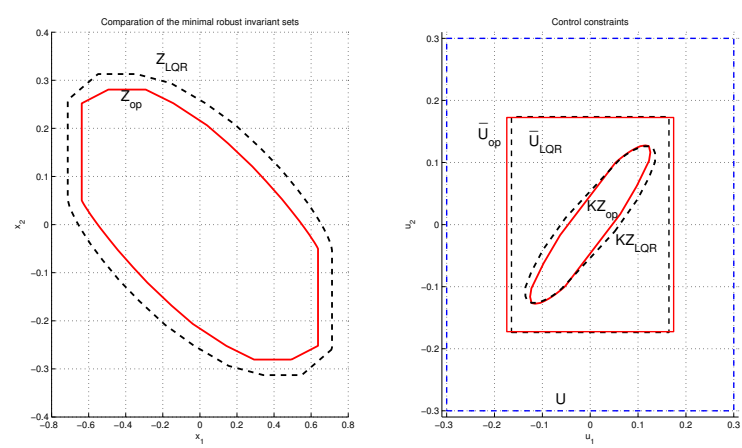

Fig. 1. Comparative of the admissible minimal robust invariant sets

In order to demonstrate the role of the parameter $\rho$, let us try to calculate the minimum robust invariant set with the minimum size, for that, pick a $\rho=1$ and consider a LQR gain with $Q=1000 * I_{2}$ and $R=1$. Figure 2 shows the RPI derived from LQR and the proposed method. It can be seen how the set $K \mathcal{Z}_{L Q R}$ is not feasible due to violates the control constraints $\left(\overline{\mathcal{U}}_{L Q R}=\mathcal{U} \ominus K \mathcal{Z}_{L Q R}\right.$ is an empty set). The proposed method provides a feasible RPI but, since $\rho=1$, the set $\overline{\mathcal{U}}_{o p}=\mathcal{U} \ominus K \mathcal{Z}_{o p}$ is quite small, so smaller is the parameter $\rho$ bigger is the robust minimal invariant set (worst disturbance rejection) but bigger is $\overline{\mathcal{U}}_{o p}$ (The nominal system has a less restrictive control constraints, so the evolution of the nominal system is faster). Thus, with the value of $\rho$ it is possible to choose between disturbance rejection and performance, as bigger is $\rho$ better disturbance rejection but worse is the performance.

\section{CONCLUSIONS}

This paper deals with the design step of the robust predictive controller for tracking (Alvarado et al. [2007]. This controller can be thought as a natural extension of predictive controllers to achieve set point tracking and has
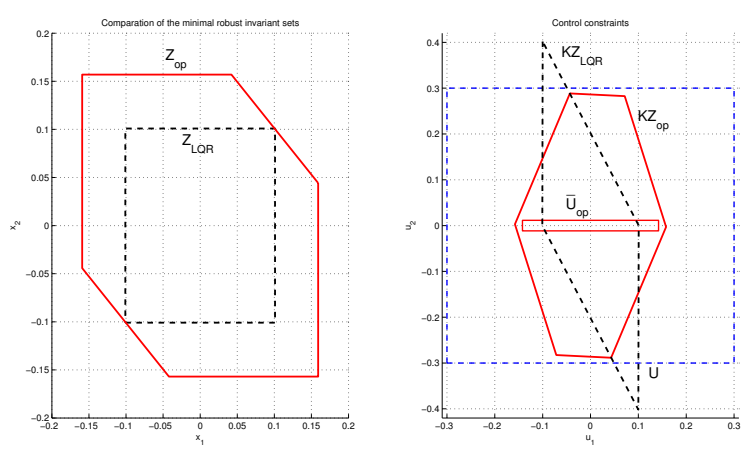

Fig. 2. Comparative of the minimal robust invariant sets some extra parameters that can be tuned to achieve the following objectives:

- Offset minimization

- Disturbance rejection by minimizing the RPI

- Guarantee of feasibility of the tube-based MPC and enlargement of the domain of attraction.

The first one depends on the tracking error cost weighting matrix $T$, which can be freely chosen (under a mild assumption) to penalize more heavily some outputs in order to minimize their offset. The last two objectives are achieved by means a LMI which can be efficiently solved.

Finally, we present a method to estimate the mRPI in the case that relative error bound is used, and the uncertainty is posed as a class of zonotopes. We also provide practical methods to derive reliable approximations in case of exact calculation is not affordable.

\section{REFERENCES}

I. Alvarado. MPC for Tracking of Constrained Linear Systems. PhD thesis, Univ of Seville., 2007.

I. Alvarado, D. Limon, T. Alamo, M. Fiacchini, and E.F. Camacho. Robust tube based MPC for tracking of piecewise constant references. In Proceedings of the $C D C$, 2007.

S. Boyd, L. E. Ghaoui, E. Feron, and V. Balakrishnan. Linear Matrix Inequalities in systems and control theory. SIAM, 1994.

I. Kolmanovsky and E. G. Gilbert. Theory and conmputation of disturbance invariant sets for discrete-time linear systems. Mathematical Problems in Engineering: Theory, Methods and Applications, 4:317-367, 1998.

D. Limon, I. Alvarado, T. Alamo, and E.F. Camacho. MPC for tracking of piece-wise constant references for constrained linear systems. Automatica, 2008. Accepted for publication.

D.Q. Mayne, M.M. Seron, and S.V. Rakovic. Robust model predictive control of constrained linear systems with bounded disturbances. Automatica, 41:219-224, 2005.

G. Pannocchia. Robust model predictive control with guaranteed setpoint tracking. Journ. of Process Control, 14:927-937, 2004.

S.V. Rakovic, E.C. Kerrigan, K.I. Kouramas, and D.Q. Mayne. Invariant approximations of the minimal robustly positively invariant sets. IEEE Transactions on Automatic Control, 50:406-410, 2005. 